\title{
ON THE MEAN VALUE OF A WEAKLY ALMOST PERIODIC FUNCTION
}

\author{
L. N. ARGABRIGHT
}

ABstract. Let $M$ denote the invariant mean on the space $W(G)$ of weakly almost periodic functions on a LCA group $G$. The purpose of this note is to show that, for each $\phi \in W(G)$,

$$
M(\phi)=\lim _{V \rightarrow\{1\}} \int_{G} \hat{f}_{V}(x) \phi(x) d x
$$

where $\{V\}$ is the system of compact neighborhoods of 1 in the character group $\Gamma$, and, for each $V, f_{V}$ is a continuous positive definite function supported in $V$ and satisfying $f_{V}(1)=1$. This answers affirmatively a question recently raised by $\mathrm{R}$. Burckel.

In his recent monograph [1] on w.a.p. functions, R. Burckel shows that functions $f_{V}$, as above, can be found so that (1) holds for every w.a.p. function $\phi$ which is the uniform limit of Fourier-Stieltjes transforms, and he asks [1, p. 81, Question 6] whether the same formula is valid for all $\phi \in W(G)$. We will show that it is.

Our proof depends on a simple fact which, while undoubtedly well known, does not seem to be readily available in the literature in the form that we need. We include it here for the sake of completeness. For $x \in G$, we use $\delta_{x} * f$ to denote the $x$-translate of a function $f$ on $G$.

LEMMA. Let $\left\{f_{\alpha}\right\}$ be a net of nonnegative functions in $L_{1}(G)$, normalized in the sense that $\int_{G} f_{\alpha}(x) d x=1$ for all $\alpha$, and satisfying the condition

(i) $\lim _{\alpha}\left\|\delta_{x} * f_{\alpha}-f_{\alpha}\right\|_{1}=0$ for all $x \in G$.

Then, for all $\phi \in W(G)$, we have

(ii) $M(\phi)=\lim _{\alpha} \int_{G} f_{\alpha}(x) \phi(x) d x$.

Proof. For each $\alpha$, regard $f_{\alpha}$ as an element of the unit ball, $\Sigma$, of the dual of $W(G)$. Since $\Sigma$ is weak*-compact, the net $\left\{f_{\alpha}\right\}$ must have at least one weak * cluster point in $\Sigma$. Furthermore, using (i), it is easy to verify that any such cluster point is an invariant mean on $W(G)$, hence must coincide with $M$. Thus $\left\{f_{\alpha}\right\}$ converges weak* to $M$, i.e. (ii) holds for all $\phi \in W(G)$.

Received by the editors March 23, 1972.

AMS 1970 subject classifications. Primary 22B99, 43A60.

Key words and phrases. Weakly almost periodic function, invariant mean.

(C) American Mathematical Society 1972 
Now let $\{V\}$ denote the system of compact neighborhoods of 1 in the character group $\Gamma$ of $G$. For each $V$, let $W$ be a compact neighborhood of 1 such that $W W^{-1} \subset V$, and let $f_{V}=\left(g_{V}\right) *\left(g_{V}\right)^{*}$ where $g_{V}$ is a continuous function vanishing outside $W$, with $\left\|g_{V}\right\|_{2}=1$.

Then $f_{V}$ is positive definite, $f_{V}$ vanishes outside $V$, and $f_{V}(1)=\left\|g_{V}\right\|_{2}^{2}=1$. Thus $\hat{f}_{V}$ is nonnegative, and $\int_{G} \hat{f}_{V}(x) d x=f_{V}(1)=1$ by the Fourier Inversion Theorem. We may (and do) require, in addition, that the $g_{V}$ 's are themselves positive definite. Thus $\hat{g}_{V}$ is nonnegative, and $\hat{f}_{V}=\left(\hat{g}_{V}\right)^{2}$.

THEOREM. $\quad M(\phi)=\lim _{V \rightarrow\{1\}} \int_{G} \hat{f}_{V}(x) \phi(x) d x$ for all $\phi \in W(G)$.

Proof. We have only to show that the net $\left\{\hat{f}_{V}\right\}$ satisfies condition (i) of the Lemma.

Let $x$ be a fixed element of $G$, and let $\varepsilon>0$. Choose $V_{0} \in\{V\}$ such that $|\gamma(x)-1|<\varepsilon / 2$ for all $\gamma \in V_{0}$. Then if $V \subset V_{0}$, we have

$$
\begin{aligned}
\left\|\delta_{x} *\left(\hat{f}_{V}\right)-\hat{f}_{V}\right\|_{1} & =\int_{G}\left|\hat{g}_{V}^{2}\left(x^{-1} y\right)-\hat{g}_{V}^{2}(y)\right| d y \\
& =\int_{G}\left(\hat{g}_{V}\left(x^{-1} y\right)+\hat{g}_{V}(y)\right)\left|\hat{g}_{V}\left(x^{-1} y\right)-\hat{g}_{V}(y)\right| d y \\
& \leqq\left\|\delta_{x} * \hat{g}_{V}+\hat{g}_{V}\right\|_{2}\left\|\delta_{x} * \hat{g}_{V}-\hat{g}_{V}\right\|_{2} \\
& =\left\|x g_{V}+g_{V}\right\|_{2}\left\|x g_{V}-g_{V}\right\|_{2} \\
& \leqq 2\left(\int_{V_{0}}|\gamma(x)-1|^{2}\left|g_{V}(\gamma)\right|^{2} d \gamma\right)^{1 / 2} \\
& \leqq 2\left(\frac{\varepsilon^{2}}{4} \int_{V_{0}}\left|g_{V}(\gamma)\right|^{2} d \gamma\right)^{1 / 2}=\varepsilon\left\|g_{V}\right\|_{2}=\varepsilon .
\end{aligned}
$$

Thus $\lim _{V \rightarrow\{1\}}\left\|\delta_{x} * \hat{f}_{V}-\hat{f}_{V}\right\|_{1}=0$, and our proof is complete.

\section{REFERENCES}

1. R. B. Burckel, Weakly almost periodic functions on semigroups, Gordon and Breach, New York, 1970. MR 41 \#8562.

Department of Mathematics, University of Nebraska, Lincoln, Nebraska 68508 